Génét. Sél. Evol., 1986, 18 (1), 63-72

\title{
Consommation alimentaire chez des poules Fayoumi et Fayoumi $\times$ Leghorn en fonction de la formation de l'œuf et de l'oviposition, avec ou sans source calcique séparée
}

\author{
M. ABD-EL-LATIF (*), A. BORDAS, P. MÉRAT \\ Laboratoire de Génétique Factorielle, \\ I.N.R.A., Centre National de Recherches Zootechniques, F 78350 Jouy-en-Josas
}

\begin{abstract}
Résumé
Des poules Fayoumi et F1 (Fayoumi $\times$ Leghorn) ont été comparées pour leurs performances de ponte, les caractéristiques de leurs oufs et pour leur consommation alimentaire en fonction de la formation d'un œuf et de l'oviposition, par des mesures journalières pendant 3 semaines, d'abord en présence d'un aliment complet, puis avec distribution séparée d'un aliment de base non enrichi en calcium et de coquillés d'huîtres. L'excès de consommation d'aliment unique (période 1) associé à la formation d'un œuf ou à l'oviposition, n'est pas différent pour les 2 types génétiques lorsqu'il est exprimé en p. 100 de la consommation journalière moyenne de la période. Dans la seconde période, la consommation de l'aliment de base n'est pas affectée par le type de jour pour l'un ou l'autre des types génétiques. Par contre, lors de la formation d'un ouf, les poules Fayoumi augmentent proportionnellement plus leur ingestion de coquilles d'huîtres que les poules de croisement. La Fayoumi semble répondre davantage, d'autre part, à l'alimentation calcique séparée quant à l'ingestion d'aliment de base, au poids moyen des œufs et au poids des coquilles par unité de surface. Indépendamment du groupe génétique, on retrouve les effets connus de la formation d'un ouf et de l'oviposition sur l'ingéré alimentaire, ainsi que ceux d'une alimentation calcique séparée sur la même variable, le poids de l'œuf et celui des coquilles.
\end{abstract}

Mots clés : Poule Fayoumi, formation de l'œuf, alimentation calcique séparée.

\section{Summary}

Feed intake of Fayoumi and Fayoumi $\times$ Leghorn hens in relation to egg formation and oviposition, with or without separate calcium feeding

The laying performance, egg traits and feed intake of Fayoumi and F1 (Fayoumi $\times$ Leghorn) hens were compared in relation to egg formation and oviposition. Daily measurements were taken over 3 weeks, first giving a complete feed and then distributing separately a basal diet containing no additional calcium and oyster shells. The excess consumption of the complete feed (period 1)

$\left(^{*}\right)$ Adresse permanente : Faculté d'Agriculture, Université d'Assiout (Egypte). 
associated with egg formation or oviposition was not different for the 2 genetic types when expressed as a percentage of average daily intake for that period. In period 2, consumption of the basal diet was not affected by the type of day in either genetic group. Conversely, when an egg was formed, the intake of oyster shells increased proportionally more in Fayoumi hens than in F1 birds. On the other hand, the Fayoumi seemed to respond more to separate calcium feeding as concerned the consumption of the basal diet, the mean egg weight and the weight of eggshells per surface unit. Independently of the genotypes, the known effects of egg formation and oviposition on feed intake were observed as well as the effects of separate calcium feeding on feed intake, egg weight and eggshell weight.

Keys words : Poultry, Fayoumi, egg formation, separate calcium feeding

\section{Introduction}

Les variations de consommation alimentaire en relation avec la formation de l'œuf et l'oviposition ont été étudiées par MoRRIS \& TAYLOR (1967), TAYLOR (1970) et WoODGush \& Horne (1970). Ces auteurs concluent que l'ingestion alimentaire est plus importante les jours où une oviposition ou surtout une ovulation a lieu que les jours sans ovulation ni oviposition. Hughes (1972) puis Mongin \& Sauveur (1974) et SAUveur \& Mongin (1974), en utilisant un aliment non enrichi en calcium et un apport séparé de coquilles d'huittres, ont mis en évidence un appétit spécifique pour le calcium lors de la formation de la coquille, appétit qui explique l'excès de consommation associé à la formation d'un cuf en présence d'un aliment unique. Les effets de l'apport d'une source séparée de calcium ont été examinés par d'autres auteurs et passés en revue par Mongin \& SAUveur (1979).

Du point de vue génétique, Mongin \& Sauveur (1974) avaient obtenu des résultats dans une population Leghorn blanche et dans un croisement $(R I R \times$ wyandotte $)$. D'autre part, Bordas \& MÉrat (1976) ont montré que, pour des poules naines (dw), la consommation alimentaire augmente relativement plus lorsqu'il y a formation d'un ouf ou oviposition que pour des poules de taille normale ( $\mathrm{Dw}$ ) provenant des mêmes familles.

Pour la race égyptienne Fayoumi, de nombreux auteurs ont noté l'épaisseur et la solidité des coquilles de ses œufs; d'autre part, Kamar et al. (1982) ont montré l'importance du calcium pendant la période de croissance du système reproductif chez les femelles. Nous avons voulu examiner, d'une part la consommation d'un aliment unique pour des poules de cette race en relation avec la formation d'un œuf ou l'oviposition, d'autre part leur réponse à l'apport d'un aliment non enrichi en calcium et d'une source séparée de cet élément. Un terme de comparaison disponible était un croisement de la même race avec une lignée de Leghorn blanches.

\section{Matériel et méthodes}

\section{A. Animaux, conditions expérimentales}

Des poussins femelles de la race égyptienne Fayoumi et d'un croisement $F_{1}\left(O^{7}\right.$ Fayoumi $\times q$ Leghorn) étaient issus de 10 coqs Fayoumi accouplés chacun à 5 poules Fayoumi et 3 poules Leghorn. Les reproducteurs appartenaient respectivement à 2 
lignées expérimentales de ces races conservées au Laboratoire. Les poussins, issus d'une éclosion pedigree le 17.2.1982 à partir d'œufs collectés pendant 3 semaines, étaient élevés au sol jusqu'à l'âge de 16 semaines, puis 93 femelles Fayoumi et 58 femelles $\mathrm{F}_{1}$ étaient mises en cages individuelles dans un même local et contrôlées pour leurs performances de ponte. Un éclairage en supplément du jour naturel assurait 14 heures de lumière par jour. L'aliment donné ad libitum pendant la période de contrôle jusqu'à l'âge de 42 semaines contenait 16 p. 100 de protéines totales, $2520 \mathrm{kcal} / \mathrm{kg}$ d'énergie métabolisable et 3,5 p. 100 de calcium. Entre les âges de 39 et 42 semaines, les poules étaient contrôlées journellement pour leur ponte, l'heure approximative de celle-ci, les caractéristiques de chaque ouf (cf. plus loin), et leur consommation d'aliment de $9 \mathrm{~h}$ à 9 h le matin.

Dans une seconde phase, entre les âges de 44 et 47 semaines, après une période d'adaptation de 2 semaines, 120 de ces mêmes poules recevaient chacune, dans 2 mangeoires séparées, un aliment identique à celui donné précédemment, sauf pour le taux de calcium, abaissé à 1 p. 100, et d'autre part des coquilles d'huîtres, contenant au moins 33 p. 100 de calcium d'après Patrick \& Shaible (1980). Comme dans la période précédente, chaque jour, outre l'heure de la ponte et des caractéristiques des oufs, la consommation d'aliment était mesurée de $9 \mathrm{~h}$ à $9 \mathrm{~h}$ et de même pour les coquilles d'huîtres.

\section{B. Mesures}

Les types de jour dans la première période étaient classés selon WooD-Gush \& Horne (1970) et Hughes (1972) : X représente un jour sans ovulation ni oviposition, L un jour avec oviposition seulement (correspondant au dernier œuf d'une série), $O$ un jour avec ovulation seulement (premier œuf d'une série), LO un jour avec à la fois ovulation et oviposition. Une série est définie comme une suite d'œufs pondus à des jours consécutifs sans interruption.

A partir des mesures de consommation journalières, on peut estimer les consommations alimentaires moyennes $\overline{\mathrm{L}}, \overline{\mathrm{O}}$ et $\overline{\mathrm{LO}}$ d'une poule respectivement pour chacun des types de jour décrits plus haut (les jours $X$, plus rares, n'étaient pas utilisés). On en tire les 2 variables $d_{O}=\overline{L O}-\bar{L}$ et $d_{L}=\overline{L O}-\bar{O}$, représentant respectivement l'excès d'ingestion alimentaire associé à la formation d'un œuf suivant une ovulation, et à l'oviposition (à laquelle s'ajoute éventuellement la fin de la calcification de la coquille). Les variables $d_{o}$ et $d_{L}$ étaient également exprimées en p. 100 de la consommation journalière moyenne de chaque type génétique sur l'ensemble de la période de 21 jours considérée.

L'heure de la ponte de chaque œuf était évaluée de façon approchée par des relevés à $9,10,11,13,14 \mathrm{~h}, 15 \mathrm{~h} 30$ et $17 \mathrm{~h}$. Il en était déduit l'intervalle de temps moyen, par individu, entre ovipositions successives dans une série. Quant aux mesures sur les oufs, celles présentées ici sont le poids, le poids de coquille et le poids de coquille ramené à la surface de l'œuf estimée en fonction du poids de celui-ci selon BonNeT \& MONGIN (1965).

Les mesures journalières dans la période d'alimentation avec apport séparé de calcium étaient les mêmes que dans la première période en ce qui concerne la consommation de l'aliment de base, et il s'y ajoutait les mesures et variables analogues pour la consommation de coquilles d'huîtres. 


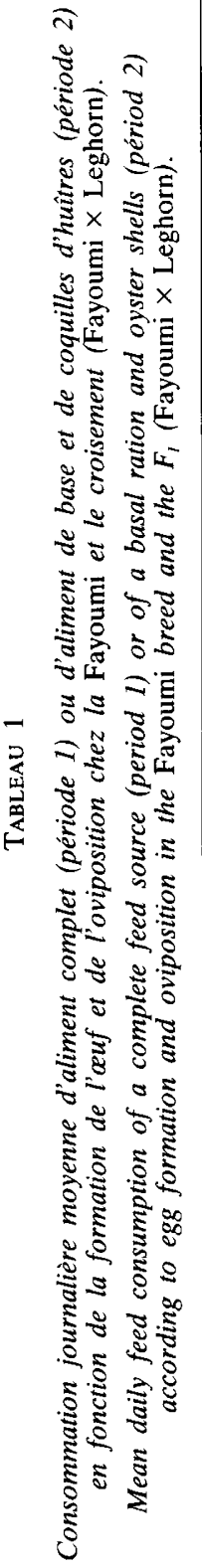

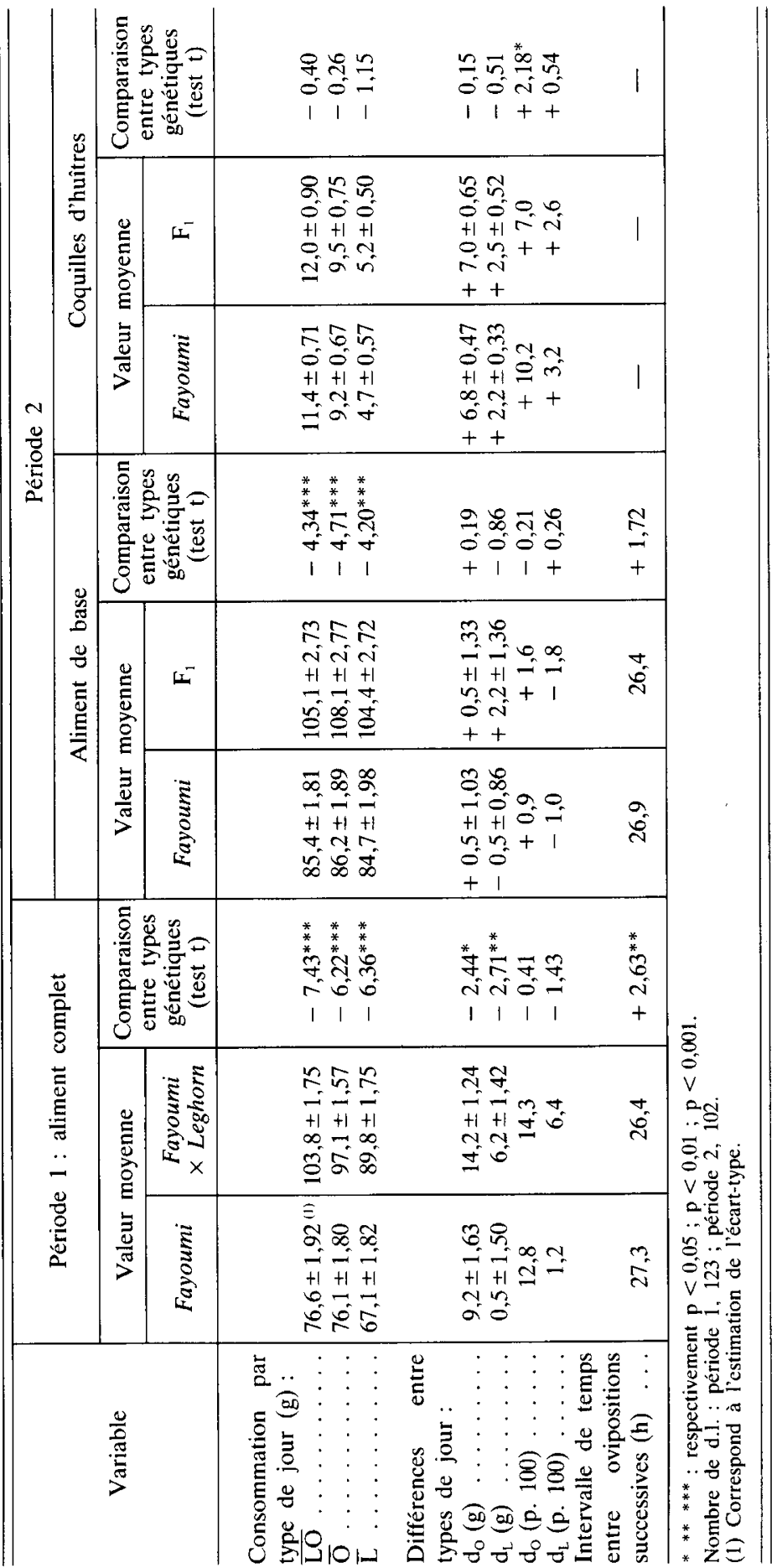




\section{Analyses statistiques}

Dans chaque période expérimentale, les 2 types génétiques, Fayoumi et croisement, sont comparés pour chacune des variables concernant la consommation journalière par un test $\mathrm{t}$ (tabl. 1). N'ont été gardées pour cette comparaison que les poules ayant pondu un nombre d'œufs permettant d'avoir au moins une estimation de consommation alimentaire pour chaque type de jour. Le nombre de poules retenues dans l'analyse, respectivement en race Fayoumi et en croisement était 72 et 53 dans la première période, 64 et 40 dans la seconde. Par suite, les effectifs par type génétique et père étaient faibles, et, respectivement, pour la Fayoumi un père dans la seconde période et pour la $F_{1}$ un père dans la période 1 et 3 dans la période 2 , n'étaient pas représentés, de sorte que la prise en compte du facteur «père » dans l'analyse des données se justifiait peu. D'autre part au tableau 2, les moyennes des variables $d_{O}$ et $d_{L}$ ont été testées d'un autre point de vue, à l'intérieur de chaque période et type génétique, par rapport à la valeur zéro (test $t$ ). Enfin, au tableau 3, à titre indicatif sur des performances non associées au type de jour, nous avons considéré, sur les poules mesurées, dans les 2 périodes, la différence individuelle d'une période à l'autre. Cette variable a d'une part été testée par rapport à la valeur zéro, et d'autre part comparée selon le type génétique par une analyse de variance à un facteur. La " période » inclut bien entendu le facteur « traitement » confondu avec un facteur «âge " (voir discussion).

\section{Résultats et discussion}

Le tableau 1 compare, pour la Fayoumi et le croisement (Fayoumi $\times$ Leghorn), les consommations moyennes d'aliment unique (période 1) pour chaque type de jour en fonction de la ponte, les variables $d_{o}$ et $d_{L}$ dérivées et l'intervalle moyen entre ovipositions successives; et, pour la période 2 , les variables analogues compte tenu de la présence d'un aliment de base et d'un apport de coquilles d'huîtres.

$\mathrm{Au}$ tableau 2 figurent les tests de la différence par rapport à la valeur zéro des variables $d_{O}$ et $d_{L}$, dans la première période avec aliment unique et dans la seconde, pour l'aliment et les coquilles d'huîtres.

Le tableau 3 met en regard les consommations moyennes (tous types de jours réunis), la ponte, le poids moyen des cufs et leur poids de coquille, absolu ou relatif à la surface estimée, dans les 2 périodes correspondant respectivement à un aliment unique et à l'alimentation avec source séparée de calcium.

La comparaison des types génétiques avec un seul aliment complet fait ressortir le fait que le croisement ingère plus d'aliment quel que soit le type de jour (tabl. 1) ce qui était aisément prévisible du fait de son poids corporel plus élevé et de sa ponte supérieure : respectivement pour la Fayoumi et la $F_{1}$. Le poids moyen à 42 semaines d'âge était 1227 et $1460 \mathrm{~g}$, le nombre d'œufs pondus jusqu'à cet âge égal à 75,1 et 88,1 , et leur poids moyen 39,4 et $48,1 \mathrm{~g}$. Les différences $d_{o}$ et $d_{L}$ en valeur absolue vont dans le même sens. Exprimées en p. 100 de la consommation journalière moyenne du groupe, $d_{o}$ et $d_{L}$ ne diffèrent pas significativement selon le type génétique. Autrement dit, la Fayoumi et le croisement modifient leur consommation de la même façon, en valeur relative, en fonction de la formation d'un ouf ou de sa ponte. Enfin, 


\section{TABLEAU 2}

Test de la différence à zéro des variables $d_{o}$ et $d_{L}$ exprimées en p. 100 dans le cas de l'aliment unique ou d'une source séparée de calcium.

Test of the deviation from zero of the $d_{o}$ and $d_{L}$ variables expressed in $p .100$ in the case of the complete feed source or with a separate calcium source.

\begin{tabular}{|c|c|c|}
\hline \multirow{2}{*}{ Variable } & \multicolumn{2}{|c|}{$\begin{array}{l}\text { Test de la différence à zéro: } \\
\text { Valeur de } t \text { et signification }\end{array}$} \\
\hline & Fayoumi & Croisement \\
\hline $\begin{array}{l}\text { Période } 1 \\
\text { Aliment unique : } \\
\mathrm{d}_{\mathrm{o}} \ldots \ldots \ldots \ldots \ldots \ldots \ldots \ldots \ldots \ldots \ldots \ldots \ldots \ldots \ldots \ldots \ldots \ldots \ldots \ldots \ldots \ldots \ldots\end{array}$ & $\begin{array}{l}5,57^{* * *} \\
0,33\end{array}$ & $\begin{array}{r}11,15^{* * *} \\
4,27^{* * *}\end{array}$ \\
\hline 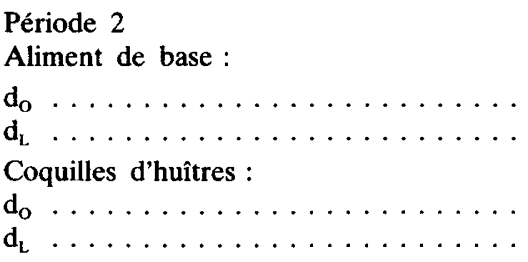 & $\begin{array}{r}0,47 \\
0,56 \\
13,81^{* * *} \\
6,55^{* * *}\end{array}$ & $\begin{array}{r}0,36 \\
-1,47 \\
10,24^{* * *} \\
4,54^{* * *}\end{array}$ \\
\hline $\begin{array}{l}* * * * * * \text { respectivement } \mathrm{p}<0,05 ; \mathrm{p}<0,01 \\
\text { Nombre de d.l.: } \\
\text { Période } 1,71 \text { pour la Fayoumi, } \\
52 \text { pour le croisement. } \\
\text { Période } 2,63 \text { pour la Fayoumi, } \\
39 \text { pour le croisement. }\end{array}$ & & \\
\hline
\end{tabular}

concordant avec la différence de ponte observée, l'intervalle de temps entre ovipositions consécutives est d'environ une heure plus long en race Fayoumi, ce qui d'ailleurs modifie légèrement la signification comparative des variables $d_{o}$ et $d_{L}$ pour cette race et ce croisement.

Indépendamment des types génétiques, on retrouve dans le tableau 1 le fait connu que la consommation alimentaire est plus élevée les jours où un œuf est en formation par rapport aux jours où il n'y a pas eu d'ovulation; cette augmentation est ici comprise entre 13 et 14 p. 100 dans l'ensemble, et la première partie du tableau 2 confirme qu'elle est très significativement différente de zéro. Par contre, l'accroissement d'ingestion d'aliment les jours de ponte est beaucoup plus faible en valeur absolue ou relative, et le tableau 2 montre qu'elle n'est significative que dans le croisement. Ceci suggère que la variable $d_{L}$ pourrait être plus élevée pour la $F_{1}$ qu'en race Fayoumi, malgré l'absence de signification du test de comparaison entre types génétiques. 


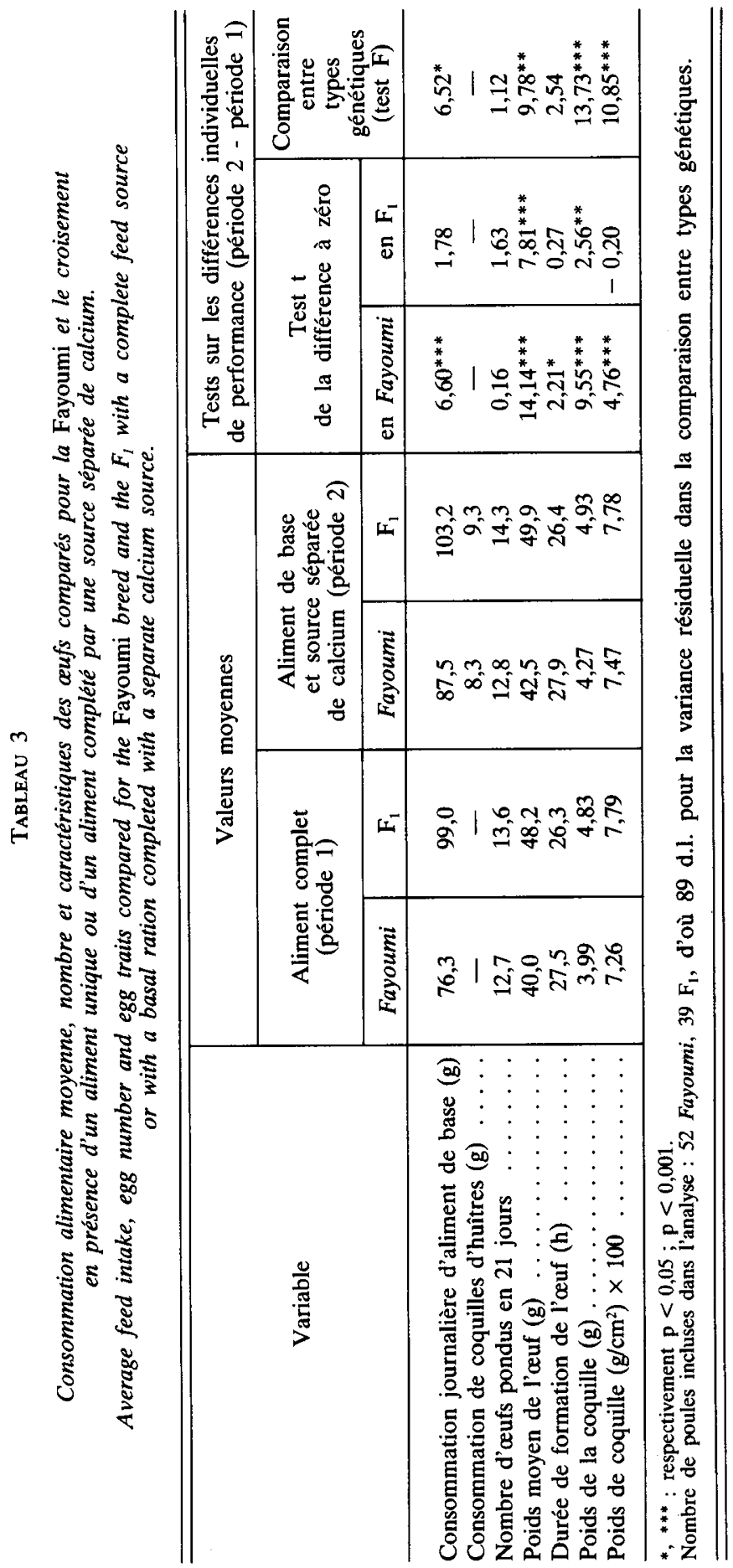


En alimentation calcique séparée, la consommation d'aliment de base du croisement est plus élevée qu'en race Fayoumi pour chaque type de jour, comme dans la période précédente et pour les mêmes raisons. Par contre, les variables dérivées $d_{o}$ et $d_{L}$, non seulement en p. 100 de la consommation journalière moyenne mais même en valeur absolue, ne présentent plus aucune différence significative entre types génétiques. Ceci va de pair avec le fait, connu par ailleurs et revérifié sur nos données au tableau 2, que pour cet aliment de base peu riche en calcium, la consommation, dans l'ensemble, ne varie pas suivant le type de jour, avec ou sans formation d'œuf. La situation est différente pour la source de calcium (coquilles d'huîtres): les poules Fayoumi en consomment pratiquement autant que les poules $F_{1}$, et ceci pour tous les types de jour ; les variables $d_{O}$ et $d_{L}$ relatives aux coquilles d'huîtres ne diffèrent pas en valeur absolue entre types génétiques, mais, en pourcentage, $d_{o}$ est plus élevé $(\mathrm{p}<0,05)$ en race Fayoumi. Cette race semble donc avoir un appétit relativement fort pour le calcium, globalement ainsi qu'en liaison avec la formation d'un œuf.

Enfin, la comparaison des consommations journalières et caractéristiques des œufs présentée au tableau 3 n'est donnée qu'à titre indicatif. Notre objectif était essentiellement de comparer 2 populations génétiquement distinctes et non de retrouver les effets déjà connus d'une alimentation calcique séparée. Aussi bien, pour des raisons matérielles, la comparaison avec un aliment unique et celle en présence d'un apport séparé de l'élément calcium ont été faites à 5 semaines d'intervalle, de sorte que les différences entre les performances dans les 2 périodes peuvent provenir non seulement du traitement alimentaire mais en partie du facteur temps. Cependant, au stade suivant le maximum de ponte où nous nous trouvions, la consommation alimentaire change peu avec le temps, et l'augmentation possible du poids moyen de l'œuf en 5 semaines est relativement minime par rapport à l'accroissement observé de l'ordre de $2,5 \mathrm{~g}$ entre la première et la seconde période. Nos données (test des variables « différences entre périodes » par rapport à la valeur zéro par type génétique au tabl. 3) concordent donc raisonnablement bien avec celles de la littérature ( $p$. ex. revues par Mongin \& SAUVEUR, 1979) selon lesquelles l'alimentation calcique séparée augmente la consommation d'aliment de base, le poids moyen des œufs et le poids des coquilles. Pour la première variable, l'examen du tableau 1 montre d'ailleurs que cette augmentation porte surtout sur les jours où il n'y a pas formation d'un œuf. Ces modifications sont d'autre part plus accentuées dans la race Fayoumi que dans son croisement, en ce qui concerne la consommation d'aliment de base, le poids de l'œuf et le poids de coquille total et par unité de surface. Les moyennes du tableau 3 et l'analyse de variance de la dernière colonne du tableau 3 le montrent et suggèrent sous la réserve indiquée plus haut quant à l'intervention du facteur âge en même temps que du traitement, une différence entre types génétiques quant à leur réponse à une alimentation calcique séparée pour ces paramètres. L'ensemble des résultats suggère donc que les poules Fayoumi répondent davantage à l'alimentation calcique séparée que le croisement, ingérant autant de calcium que ce dernier malgré leur petite taille, avec répercussion sur le poids des coquilles. Ceci est sans doute à rapprocher de l'épaisseur et de la solidité bien connues de la coquille des œufs dans cette race, mais le mode de contrôle génétique de l'appétit calcique et la base physiologique des différences observées ici restent à déterminer. 


\section{Références bibliographiques}

Bonnet Y., Mongin P., 1965. Mesure de la surface de l'œuf. Ann. Zootech., 14, 311-317.

Bordas A., Mérat P., 1976. Effect of laying on food and water intake in dwarf and normal hens. Brit. Poult. Sci., 17, 415-426.

Hughes B.O., 1972. A circadian rhythm of calcium intake in the domestic fowl. Brit. Poult. Sci., 13, $485-493$.

Kamar G.A.R., El-Shaffie M.M.H., El-Nadi M.M., Samy M.S., 1982. Weight changes in the reproductive organs of the Fayoumi and Rhode-Island hens and its relation to dietary supplemented calcium levels. Assiut Vet. Med. J., 10, 43-51.

Mongin P., Sauveur B., 1974. Voluntary food and calcium intake by the laying hen. Brit. Poult. Sci., 15, 349-359.

Mongin P., Sauveur B., 1979. The specific calcium appetite of the domestic fowl. In : Boorman K.N., Freeman B.M. (ed), Food intake regulation in poultry, 171-189, British Poultry Science, Edinburgh.

MorRis B.A., TAYLOR T.G., 1967. The daily food consumption of laying hens in relation to egg formation. Brit. Poult. Sci., 8, 251-257.

Patrick H., Schaible P.J., 1980. Poultry feeds and nutrition, 668 pp., Avi Publishing Co, Westport, Conn.

Sauveur B., Mongin P., 1974. Effect of time limited calcium meal upon food and calcium ingestion and egg quality. Brit. Poult. Sci., 15, 305-313.

Taylor T.G., 1970. The role of the skeleton in eggshell formation. Ann. Biol. Anim. Bioch. Biophys., 10, 84-91.

Wood-Gush O.G., Horne A.R., 1970. The effect of egg formation and laying on the food and water intake of Brown Leghorn hens. Brit. Poult. Sci., 11, 459-466. 